\title{
Quand une haute personnalité politique s'engage pour la «new public health»
}

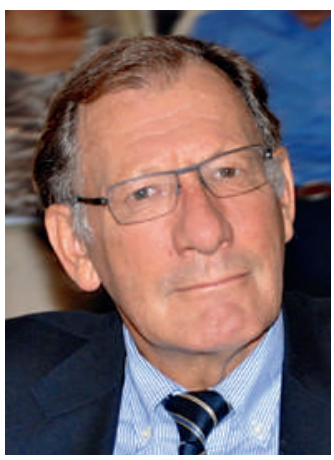

Jean Martin
J'ai été frappé de lire plusieurs articles étoffés sur Michael Bloomberg, ingénieur et manager-financier devenu très riche (31 milliards de dollars), au moment où il termine à fin 2013 son mandat de 12 ans de maire de New York [1, 2]. Le plus important, d'un point de vue de politique de santé, est celui d'une figure respectée du droit de la santé aux Etats-Unis, Lawrence Gostin de l'Université Georgetown [3].

A les lire, Bloomberg est de ces personnalités hyperactives et «hyper-achieving», s'engageant avec succès dans de multiples domaines. Après son départ de la mairie, il entend développer des projets sur plusieurs continents - y compris en Europe en vue d'y améliorer la gouvernance des grandes villes. Mais j'en parle ici pour ses réalisations en matière de santé. On connaît les problèmes majeurs aux Etats-Unis en rapport avec l'obésité, liée à des non-foods bourrées de calories (y compris boissons) et à la sédentarité. Bloomberg a voulu éviter que ses concitoyens ne soient quotidiennement poussés à mal manger; il a ainsi décrété un ban sur les graisses trans, a obligé les restaurants à déclarer sur leurs menus la teneur calorique, a lancé un partenariat public-privé pour réduire la consommation de sel. Et (surprenant au premier abord), il a voulu limiter la contenance des emballages de «soft drinks» - pour éviter l'absorption de trop de sucre. Cette mesure toutefois a été contrée légalement et les tribunaux ont jugé que le «Board of Health» de la ville n'avait pas la compétence d'imposer une telle limite.

Ce dernier exemple illustre que les secteurs industriels et commerciaux concernés ne sont pas prêts à se laisser faire... Jouant sur toute la gamme des «valeurs américaines», ils attaquent des mesures qui ont pourtant un vrai potentiel bénéfique, pour la santé des gens et pour la facture médico-sanitaire. Critiquant les limites promulguées comme des atteintes à la liberté de chacun (de se nuire...) et présentant le maire comme une meddling nanny (nounou importune) s'ingérant indûment dans la vie des personnes. En passant, on note que c'est par ces invocations d'une liberté individuelle qui serait intouchable que sont bloquées, ou pour le moins freinées, aux EtatsUnis en ce moment, des avancées de santé publique.

Tableau comparable pour le tabagisme. Bloomberg a introduit à son arrivée l'interdiction de fumer dans des locaux puis dans certains espaces extérieurs; il a augmenté les taxes sur les cigarettes et soutient les efforts de l'OMS au niveau mondial; mais c'est sans succès qu'il a cherché a introduire des restrictions quant au marketing du tabac. Il a aussi amélioré pour les New-Yorkais les possibilités de prendre de l'exercice (vélo, jogging). A noter qu'il n'a pas pour autant diminué les efforts de salubrité publique, pourrait-on dire, dans d'autres domaines comme la sécurité (à quoi son prédécesseur Giuliani avait utilement œuvré déjà). Il est engagé pour le contrôle des armes à feu et, autre enjeu, pour faire reconnaître le problème du changement climatique.

Comme déjà dit, les secteurs qui voient leurs intérêts pécuniaires touchés montrent rarement le bon sens et le souci du bien public qui les feraient accepter de ne plus mettre sur le marché des produits nuisibles. Tous les arguments sont bons dans ce combat. Il est vrai par ailleurs que la question de poser des limites, à la liberté économique notamment, doit être attentivement considérée. Si la loi définit ce qui est admissible et ce qui ne l'est pas, son rôle n'est pas pour autant de dire ce qui est bien. Il est facile en effet de faire une longue liste de produits dont on peut licitement faire commerce et de comportements encouragés par la publicité qui sont au détriment de la santé et du bien-être des personnes. L'Etat n'a pas à faire la morale, des citoyens capables de discernement ont le droit de mener leur vie comme ils l'entendent (sous réserve de tort fait à autrui). Reste toutefois à savoir, question majeure, si les comportements qu'ils adoptent le sont véritablement librement: les moyens de pression divers, le matraquage publicitaire et le marketing, souvent attrayants, disposent de bien plus de moyens financiers et d'influence que les institutions médico-sanitaires et la société civile qui promeuvent des modes de vie sains.

Pour résumer: encourageant de voir un généraliste de la politique de très haut niveau (et qui n'est certainement pas de gauche) s'engager carrément pour que les intérêts de santé prévalent sur les gains commerciaux à court terme. Selon L. Gostin, on a là une exemple de «new public health»: une stratégie qui veut tenir compte simultanément - et dans une optique à long terme - des impacts (positifs ou négatifs) sur la santé de l'action des secteurs publics ainsi que de l'économie en général. «Les gouvernements», dit-il, «doivent être tenus pour responsables de la santé de leur population.»

Jean Martin,

médecin de santé publique et membre de la rédaction 